\title{
Statistical Analysis of the Tensile Strength of Carbon Nanotubes
}

\author{
Chunsheng Lu \\ Department of Mechanical Engineering, Curtin University of Technology, Perth, WA 6845, Australia \\ C.Lu@curtin.edu.au
}

Keywords: Carbon nanotube; Tensile strength; Weibull statistics; Lognormal distribution; Minimum information criterion.

\begin{abstract}
Two available strength data sets of single-walled and multi-walled carbon nanotubes are analysed, and the effects of sample sizes on their tensile strengths are investigated. A minimum information criterion is applied to determine the optimal strength distribution. The results show that, in contrast to a two-parameter Weibull distribution, lognormal distribution seems to be a more suitable choice. A simple extrapolation of classical Weibull statistics to nanoscales may result in overestimation on the tensile strength of carbon nanotubes.
\end{abstract}

\section{Introduction}

Carbon exists in various polymorphic forms such as graphite and diamond. Carbon nanotubes are a new form of carbon that consists of graphite sheets, rolled into hollow tubes and capped with $\mathrm{C}_{60}$ fullerene hemispheres on both ends. In general, there are two types of carbon nanotubes: single-walled carbon nanotubes (SWCNTs) with diameters of $0.5-2 \mathrm{~nm}$ and multiple-walled carbon nanotubes (MWCNTs) with diameters of 2-50 nm [1-3]. These nanotubes have unique and promising physico-mechanical properties such as low density, superior electrical/thermal conductivity, extremely strong stiffness and strength, and in addition, small but relatively ductile with fracture strain of $\sim 5 \%$. Combined with huge aspect (length-to-diameter) ratios, carbon nanotubes are considered as an ideal reinforcer in composite materials [3]. It is not surprising that the strength of carbon nanotubes has been of great interest; however, their values have remained elusive both experimentally and theoretically.

The small size and special microstrucure of carbon nanotubes make them entirely different from carbon fibres. It is known that the scattered strengths of carbon fibres with diameters on the scale of micrometres can be well described in terms of Weibull statistics [4,5]. As materials or specimens approach nanoscales, however, the principal assumptions such as continuum in linear elastic fracture mechanics are unsuitable [6,7]. Similar problems also appear in statistical analysis by using classical Weibull statistics because there are only a few rather than numerous defects in nanostructured materials such as carbon nanotubes. In addition, nano-defects are completely different from those like cracks in continuum mechanics. Thus, the effectiveness of Weibull statistics at nanoscales is often in question and there is still no consensus on its validity [8-11]. The recent work showed that, just based on post-mortem data analysis, it seems difficult to identify the feasibility of Weibull statistics at micro- and nano-scales, and a simple extrapolation may result in overestimation on the strength of a tested material, or more seriously, a misunderstanding on its intrinsic fracture mechanism $[12,13]$. In other words, to correctly estimate the strength of carbon nanotubes as well as other nanostructured materials, a critical statistical analysis on their strength data obtained from tests is needed.

In this paper, two available data sets for the tensile strengths of SWCNTs and MWCNTs are comprehensively analysed. The influences of specimen sizes such as lengths and/or diameters of SWCNTs and MWCNTs on their tensile strengths are investigated. In order to determine the optimal distribution for a given experimental data set, a simple method in terms of a minimum information criterion is applied. Finally, a fundamental question on the nature of nano-defects in carbon nanotubes is discussed. 


\section{Strength Data of Carbon Nanotubes}

Early measurements on the tensile strength of carbon nanotubes were done indirectly through their composites, which led to an unexpected low tensile strength of $\sim 1.2 \mathrm{GPa}$ [14]. With advances in instrumentation and technique, a more direct strength measurement on carbon nanotubes has been possible in recent years, and a few strength data sets have been obtained [8,9,15-17]. Table 1 lists two strength data sets of SWCNTs and MWCNTs, measured with a "nanostressing stage" located within a scanning electron microscope [16,17]. Also listed are geometric sizes such as diameters and lengths of carbon nanotubes. In Table 1 , the breaking strength $\sigma_{e}$ was calculated by considering all SWCNTs in a rope to be carrying the applied load, and the breaking strength $\sigma_{p}$ was calculated by considering only nanotubes in the perimeter of the rope to be carrying the applied load. Further analysis showed that $\sigma_{p}$ gives a more reasonable result for SWCNTs [16]. As to MWCNTs, the out shell was proven to be carrying the applied load [17].

Table 1. Experimental results of the tensile strengths of SWCNTs and MWCNTs and their geometric sizes $[16,17]$, where $D_{s}$ and $D_{m}$ indicate the diameter of the rope of SWCNTs and the outer diameter of MWCNTs, respectively.

\begin{tabular}{|c|ccc|ccc|}
\hline \multirow{3}{*}{ Sample } & \multicolumn{3}{|c|}{ SWCNTs } & \multicolumn{3}{c|}{ MWCNTs } \\
\cline { 2 - 7 } & $\begin{array}{c}\text { Diameter } \\
D_{s}(\mathrm{~nm})\end{array}$ & $\begin{array}{c}\text { Strength } \\
\sigma_{e}(\mathrm{GPa})\end{array}$ & $\begin{array}{c}\text { Strength } \\
\sigma_{p}(\mathrm{GPa})\end{array}$ & $\begin{array}{c}\text { Diameter } \\
D_{m}(\mathrm{~nm})\end{array}$ & $\begin{array}{c}\text { Length } \\
L(\mu \mathrm{m})\end{array}$ & $\begin{array}{c}\text { Strength } \\
\sigma_{s}(\mathrm{GPa})\end{array}$ \\
\hline 1 & 20 & 11 & 33 & 28.0 & 4.10 & 11 \\
2 & 40 & 9 & 52 & 28.0 & 6.40 & 12 \\
3 & 21 & 4 & 13 & 19.0 & 3.03 & 18 \\
4 & 38 & 8 & 48 & 31.0 & 1.10 & 18 \\
5 & 35 & 8 & 43 & 28.0 & 5.70 & 19 \\
6 & 27 & 11 & 45 & 19.0 & 6.50 & 20 \\
7 & 39 & 5 & 32 & 18.5 & 4.61 & 20 \\
8 & 34 & 3 & 16 & 33.0 & 10.99 & 21 \\
9 & 41 & 6 & 37 & 28.0 & 3.60 & 24 \\
10 & 23 & 5 & 17 & 36.0 & 1.80 & 24 \\
11 & 34 & 5 & 29 & 29.0 & 5.70 & 26 \\
12 & 23 & 7 & 23 & 13.0 & 2.92 & 28 \\
13 & 23 & 4 & 15 & 40.0 & 3.50 & 34 \\
14 & 19 & 7 & 22 & 22.0 & 6.67 & 35 \\
15 & 23 & 7 & 25 & 24.0 & 1.04 & 37 \\
16 & & & & 24.0 & 2.33 & 37 \\
17 & & & & 22.0 & 6.04 & 39 \\
18 & & & & 20.0 & 8.20 & 43 \\
19 & & & & & 6.87 & 63 \\
\hline
\end{tabular}

The mean values of the tensile strengths $\left(\sigma_{p}\right.$ and $\sigma_{s}$ ) of SWCNTs and MWCNTs in Table 1 are 30 $\mathrm{GPa}$ and $28 \mathrm{GPa}$, respectively. These values approximately lie on the same order of magnitudes with the theoretically predicted strength of $E / 10=100 \mathrm{GPa}$, where $E$ is Young's modulus of carbon nanotubes which is equal to that of graphite in-plane ( 1000 GPa) [7-9]. In contrast to carbon fibers, there seems no obvious tendency of the strengths of carbon nanotubes that vary with their diameters or lengths although the scattered values were observed. The potential reasons for the discrepancy of measured strengths of carbon nanotubes from their theoretical value will be the focus in the following statistical analysis. 


\section{Statistical Analysis}

Based on the weakest link model and a simple empirical function, the probability of failure $P_{f}$ for a specimen of volume $V$ under uniaxial stress $\sigma$ can be expressed as

$$
P_{f}(\sigma)=1-\exp \left[-\frac{V}{V_{0}}\left(\frac{\sigma}{\sigma_{0}}\right)^{m}\right] \text {, }
$$

where $V_{0}$ is a reference volume; $\sigma_{0}$ and $m$ are a normalized stress and the Weilbull modulus, respectively [4,5]. Given that the defect density is independent of the size of a specimen, the number of defects in the specimen (or the probability of containing a critical defect) is proportional to its volume. That is, the strength of a specimen decreases with increasing its size. According to Eq. (1), we have

$$
\sigma\left(V_{2}\right)=\sigma\left(V_{1}\right)\left(\frac{V_{1}}{V_{2}}\right)^{1 / m}
$$

where $V_{1}$ and $V_{2}$ are the effective volumes of two specimens, and in general, $\sigma(V) \sim V^{-1 / m}$, where $V$ is an effective volume or length (diameter). This provides us an alternative method to check the feasibility of classical Weibull statistics at nanoscales [12,13]. Relative to directly fitting Eq. (1) to the strength data of carbon nanotubes, the latter is more intuitive and easier to carry out. For example, in the case of SWCNTs under a tensile load, strengths (either $\sigma_{e}$ or $\sigma_{p}$ ) versus their diameters $D_{s}$ are shown in Fig. 1(a). Similarly, the dependence of the strengths of MWCNTs under a (nearly) uniform tension on their effective volumes is shown in Fig. 1(b). Here, the effective volumes of MWCNTs were calculated by $V=\pi D_{m} L t$, where $D_{m}, L$, and $t(\sim 0.34 \mathrm{~nm})$ are its outer diameter, length, and thickness, respectively. From Fig. 1, it is obvious that there is no size effect in both SWCNTs and MWCNTs as expected. In other words, the Weibull distribution cannot well describe the nature of the tensile strengths of carbon nanotubes. The similar conclusion was obtained in [10] by using the linear regression of Eq. (1) to these strength data.
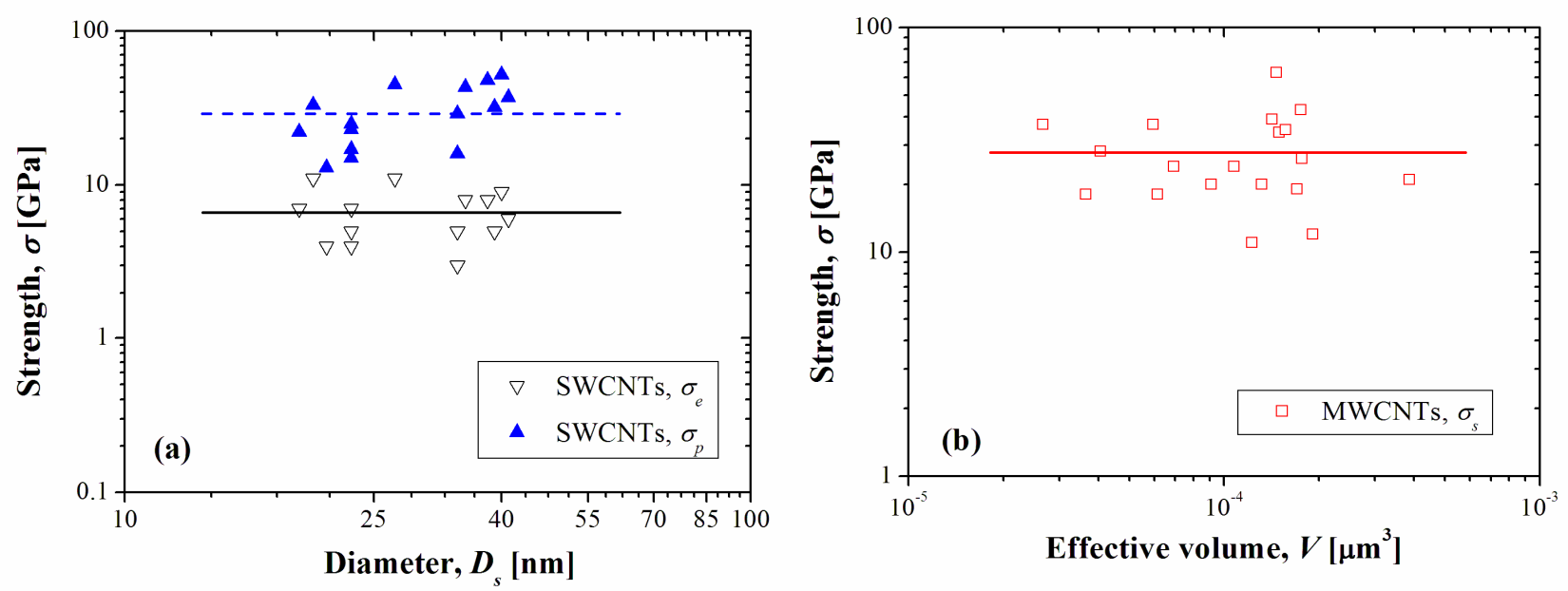

Fig. 1. (a) Tensile strengths of SWCNTs versus their diameters and (b) tensile strengths of MWCNTs versus their effective volumes, where solid and dashed lines indicate their arithmetic mean strengths, 6.7 GPa, $30 \mathrm{GPa}$ for SWCNTs, and $28 \mathrm{GPa}$ for MWCNTs, respectively.

However, it is worth noting that, for the sake of simplicity, the size influence of carbon nanotubes on their strengths is usually ignored in the statistical analysis as did in recent studies [8,9]. It seems to 
be a reasonable approximation considering the difference of diameters or lengths in 15 SWCNTs and 19 MWCNTs is less than one order of magnitude and also the scatter of strength data as shown in Fig. 1. Thus, Eq. (1) may be simplified and rewritten as

$$
P_{f}(\sigma)=1-\exp \left[-\left(\frac{\sigma}{\sigma_{0}}\right)^{m}\right] \text {. }
$$

Fitting the strength data by using the maximum likelihood method, we had two parameters: $\sigma_{0}=$ $7.47 \mathrm{GPa}, m=3.08$ (measured by $\sigma_{e}$ ); $\sigma_{0}=33.89 \mathrm{GPa}, m=2.67$ (measured by $\sigma_{p}$ ) for SWCNTs, and $\sigma_{0}=31.48 \mathrm{GPa}, m=2.40$ for MWCNTs. As shown in Fig. 2, it is of interest to see that Eq. (2) fits all the data sets well. Additionally, a small Weibull modulus $(\sim 3)$ was obtained. As we know, the smaller the Weibull modulus, the wider variability is their tensile strengths. Intuitively, this seems consistent with what is shown in Fig. 1, however, the conclusion about the size effect of strengths is just opposite to the assumption introduced in Eq. (2).

In view of the strength statistics, a two-parameter Weibull distribution in Eq. (3) should be considered as an empirical one on an equal footing with other possible candidates such as normal and lognormal distributions [4,18]. Next, additional analysis on the strength data of carbon nanotubes will be done in order to have a better understanding on physical implications of the scattered strength data, as shown in Fig. 1. For a normal distribution, its probability density function, $p_{f}(\sigma)=d P_{f}(\sigma) / d \sigma$, is

$$
p_{f}(\sigma)=\frac{1}{\sqrt{2 \pi} \alpha} \exp \left[-\frac{(\sigma-\mu)^{2}}{2 \alpha^{2}}\right] \text {, }
$$

where $\mu$ and $\alpha$ are the mean value and standard deviation, respectively. Similarly, for a lognormal distribution, its probability density function can be obtained just by replacing $\sigma$ in Eq. (4) with $\ln \sigma$, but $\mu$ and $\alpha$ in a lognormal distribution indicate the geometric mean value and standard deviation, respectively. Here, a major difference lies in the effect of independent factors that are additive or multiplicative. The two parameters in normal and lognormal distributions can be easily determined by using the maximum likelihood method [12].

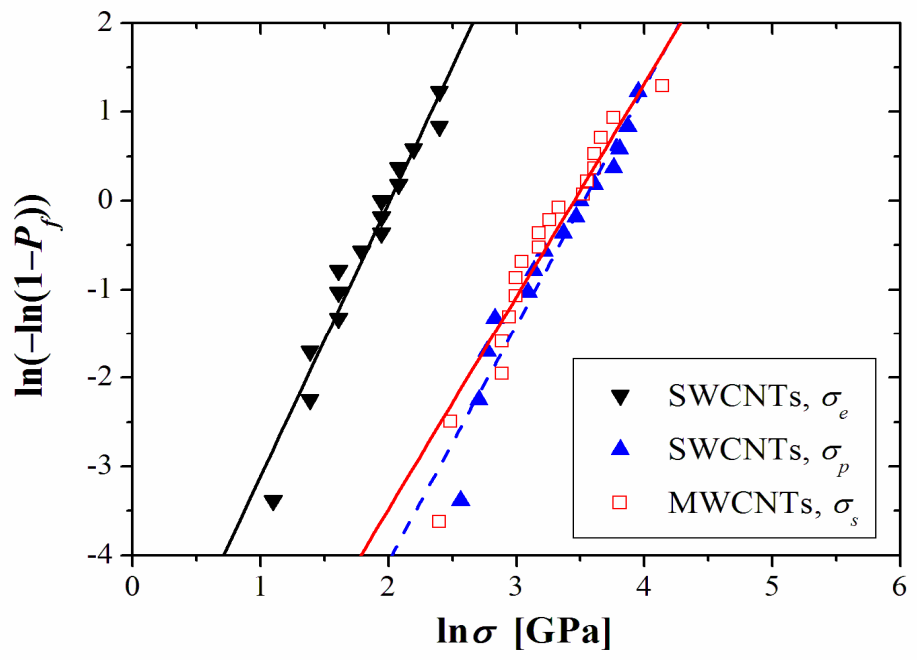

Fig. 2. Weibull distributions in a log-log plot of $\ln [1-F(\sigma)]^{-1}$ versus $\sigma$, where the calculated Weibull moduli are 3.08 and 2.67 for SWCNTs measured by $\sigma_{p}$ and $\sigma_{p}$, and 2.40 for MWCNTs, respectively. 
To determine which distribution fits these strength data sets better, a minimum (or Akaike) information criterion (AIC) is used, which is defined as, $\mathrm{AIC}=-2 \ln \hat{L}+2 k$, where $\ln \hat{L}$ is the maximum log-likelihood of a given model (or distribution) and $k$ is the number of independent parameters to be fitted in the model [19]. This provides a useful measure of the relative effectiveness of models with various parameters [20,21]. In typical cases, the difference between two distributions which would be significant at around the 5\% confidence level corresponds to the difference in AIC values of around 1.5-2.

\section{Results and Discussion}

Table 2 lists the AIC values obtained by fitting three (i.e., Weibull, normal, and lognormal) distributions to the strength data sets of carbon nanotubes in Table 1. The results show that, in the case of MWCNTs, a lognormal distribution fits the data better than a Weibull distribution because the difference of their AIC values is substantial, that is, $\Delta \mathrm{AIC}=\left|\mathrm{AIC}_{\mathrm{ln}}-\mathrm{AIC}_{\mathrm{w}}\right|>2$. But, in the case of SWCNTs, the differences between their AIC values are not large enough to distinguish which distribution is better. Considering the unrealistic assumption (i.e. size effect) implied in Eq. (3) and the unphysical assumption in a normal distribution (where a negative strength can be chosen), it seems that, in all the three data sets, a lognormal distribution is an optimal choice.

Table 2. AIC values calculated by using Weibull, normal, and lognormal distributions, where $N$ is the number of samples.

\begin{tabular}{|c|rrrrr|}
\hline Sample & $N$ & $\mathrm{AIC}_{\mathrm{w}}$ & $\mathrm{AIC}_{\mathrm{n}}$ & $\mathrm{AIC}_{\ln }$ & \multicolumn{1}{c|}{$\Delta \mathrm{AIC}$} \\
\hline SWCNTs $\left(\sigma_{e}\right)$ & 15 & 71.78 & 72.24 & 71.46 & -0.32 \\
SWCNTs $\left(\sigma_{p}\right)$ & 15 & 120.90 & 121.89 & 120.96 & 0.06 \\
MWCNTs $\left(\sigma_{s}\right)$ & 19 & 151.21 & 153.10 & 148.74 & -2.47 \\
\hline
\end{tabular}

According to the above analysis, we can give a new explanation on the scatter of tensile strengths of carbon nanotudes. An optimal lognormal distribution implies that there be some characteristic values such as mean strengths in quasi-perfect carbon nanotubes (see Fig. 1). The value of a mean strength is dependent on the collective (multiplicative) interaction of independent (or quantized) factors (not limited to flaws in a traditional sense) rather than a critical one like crack that triggers the failure as supposed in classical Weibull statistics [22,23]. Recently, nanoscale Weibull statistics, a modification of classical Weibull statistics, was proposed by Pugno and Rouff [10] in terms of their early work on quantized fracture mechanics [7]. In nanoscale Weibull statistics, a similar formula as Eq. (3) was derived, however, the stress $\sigma$ is a mean value rather than an individual one in a classic Weibull distribution [24]. This is consistent with our numerical analysis. That is, there seems to be a new kind of sensitiveness of strengths to flaws at nanoscales, and more generally, the strength of a nanostructured material may be insensitive to its critical defect $[25,26]$.

\section{Summary}

Two available strength data sets of carbon nanotubes have been investigated. A minimum information criterion can be applied to determine the optimal strength distribution for a given strength data set. The results show that an uncritical extension of classic Weibull statistics may cause overestimation on the intrinsic strength of a small scale material, and even a misunderstanding on its underlying fracture mechanism. Further statistical analysis indicates that a lognormal distribution is optimal, and there is a characteristic strength in carbon nanotubes. Finally, it is worth noting that these conclusions are just based on post-mortem data analysis. Thus, more work is needed to check their universality to materials at nanoscales. 


\section{Acknowledgements}

This work has been supported by the Curtin Internal Research Grants (IRGs) and Curtin Research Fellowship.

\section{References}

[1] S. Iijima: Nature Vol. 354, (1991), p. 56.

[2] S. Iijima: Physica B Vol. 323, (2002), p. 1.

[3] P.M. Ajayan and J.M. Tour: Nature Vol. 447, (2007), p. 1066.

[4] W. Weibull: J. Appl. Mech.-Trans. ASME Vol. 18, (1951), p. 293.

[5] B.R. Lawn: Fracture of Brittle Solids (Cambridge University Press, Cambridge, 1993).

[6] A.A. Griffith: Philos. Trans. R. Soc. London A Vol. 221, (1920), p. 163.

[7] N.M. Pugno and R.S. Ruoff: Philos. Mag. Vol. 84, (2004), p. 2829.

[8] A.H. Barber, R. Andrews, L.S. Schadler and H.D. Wagner: Appl. Phys. Lett. Vol. 87, (2005), 203106.

[9] A.H. Barber, I. Kaplan-Ashiri, S.R. Cohen, R. Tenne and H.D. Wagner: Compos. Sci. Technol. Vol. 65, (2005), p. 2380.

[10] N.M. Pugno and R.S. Ruoff: J. Appl. Phys. Vol. 99, (2006), 024301.

[11] M. Yang, V. Koutsos and M. Zaiser: Nanotechnology Vol. 18, (2007), 155708.

[12] C. Lu, R. Danzer and F.D. Fischer: Phys. Rev. E Vol. 65, (2002), 067102.

[13] C. Lu, R. Danzer and F.D. Fischer: J. Eur. Ceram. Soc. Vol. 24, (2004), p. 3643.

[14] H.W. Zhu, C.L. Xu, D.H. Wu, B.Q. Wei, R. Vajtai and P.M. Ajayan: Science Vol. 296, (2002), p. 884.

[15] E.W. Wong, P.E. Sheehan and C.M. Lieber: Science Vol. 277, (1997), p. 1971.

[16] M.F. Yu, B.S. Files, S. Arepalli and R.S. Ruoff: Phys. Rev. Lett. Vol. 84, (2000), p. 5552.

[17] M.F. Yu, O. Lourie, M.J. Dyer, K. Moloni, T.F. Kelly and R.S. Ruoff: Science Vol. 287, (2000), p. 637.

[18] E.J. Gumbel: Statistics of Extremes (Columbia University Press, New York, 1958).

[19] H. Akaike: IEEE Trans. Autom. Control Vol. 19, (1974), p. 716.

[20] C. Lu, D. Vere-Jones and H. Takayasu: Phys. Rev. Lett. Vol. 82, (1999), p. 347.

[21] C. Lu, Y.-W. Mai and Y.G. Shen: Phys. Rev. E Vol. 72, (2005), 027101.

[22] R.S. Ruoff: Proc. Natl. Acad. Sci. USA Vol. 103, (2006), p. 6779.

[23] T. Dumitrica, M. Hua and B.I. Yakobson: Proc. Natl. Acad. Sci. USA Vol. 103, (2006), p. 6105.

[24] C. Lu: Appl. Phys. Lett. (2007), submitted.

[25] A. Carpinteri and N. Pugno: Nat. Mater. Vol. 4, (2005), p. 421.

[26] H. Gao, B.H. Ji, I.L. Jager, E. Arzt and P. Fratzl: Proc. Natl. Acad. Sci. USA Vol. 100, (2003), p. 5597. 